\title{
Neurologic Complications of Cancer
}

\section{Lisa M. DeAngelis and Jerome B. Posner, Oxford University Press, Oxford, NY, 2008, 2nd edition, \$125, ISBN: 978-0-19-536674-7}

\author{
Eelco F. M. Wijdicks
}

Published online: 11 November 2008

(C) Humana Press Inc. 2008

Cancer is so pervasive in medicine and critically illness that this book is an essential reference for any hospitalist or intensivist. Complications of cancer may lead to acute critical illness from progressing disease or complications of treatment. Many patients may have prolonged stays in surgical and medical intensive care units, and a neurologic manifestation may prompt a consult. In tertiary centersnext to neurooncologists-neurointensivists are often asked to provide consultative services in the ICU. Patients with cancer may also be admitted to Neurosciences ICU with a progressive paraneoplastic syndrome or solitary metastasis resulting in a flurry of seizures or status epilepticus.

This new edition by DeAngelis and Posner is invaluable not only in understanding the pathophysiology of this established field—-both authors are recognized pioneers- but also provides specific treatment options in handling these unfortunate complications. Problems such as intractable pain, spinal cord compression, seizures are ubiquitous but are discussed in minute detail.

There is a superb chapter on delirium and nutritional complications of cancer patients but there are also equally strong chapters on CNS infections and vascular disorders. Its strong points are its rich content, the clear discussions on pathophysiology, supportive care, and management of complex complications. A weak point is the surprising absence of guidance to patients and families and end of life care.

There is very little in this book does not affect the practice of the neurointensivist. Now thoroughly updated and modernized, this is an enormously worthwhile book.

E. F. M. Wijdicks ( $\square)$

Mayo Clinic, Rochester, MN, USA

e-mail: wijde@mayo.edu 